\title{
How to Identify Tar in Heavy Oil Reservoirs
}

\author{
Naim Al-Jabari, Rafael Vasquez Halliburton
}

To calculate reserves in heavy oil reservoirs, the amount of possible tar needs to be accurately estimated. This presentation discusses how Halliburton's tar mapping technique was developed based on combining experience from different product lines over the years. The mapping technique combines successful NMR based tar identification techniques, heavy oil sampling, stimulation and chemostratigraphy.

Historically tar identification first attempts were made initially using NMR based techniques. The assumption made was that any deficit porosity between NMR porosity and Density-Neutron porosity is tar, which is true in light and medium oil reservoirs. This simple approach was very successful in Latin America where NMR based tar interpretation was in agreement with side wall cores. However, in heavy oil reservoirs, tar identification based on NMR is more complicated as both tar and heavy oil NMR's result in same deficit porosity effect. Development of second generation of NMR based interpretation technique was driven not to identify tar but to identify sweet spots in heavy oil that could be produced using cold production techniques. The second generation of NMR based interpretation technique was made by redistributing NMR measured volumes using classical well interpretation to classify the heavy oil signal to three parts: a movable part, a partially movable, and a non-movable part. To support this classification, assessment of mobility by comparing flush zone saturation from NMR with virgin zone saturation was used. This was found to be more representative of mobility than classical resistivity based flush zone saturation. Especially in the case of heavy oil reservoirs where resistivity based saturation in the flush zone will underestimate fluid mobility. To further confirm the NMR interpretation and to ensure the presence of a sweet spot of mobile oil within heavy oil, downhole fluid sampling was required. Several innovative tool modifications and developments were made to be able to sample successfully in heavy oil reservoirs. The latest was to address sampling heavy oil in shallow unconsolidated formations. The latter development establishes an accurate methodology to identify the movable oil part in heavy oil but not tar. To separate the nonmovable part into producible heavy oil and tar that cannot be produced, downhole wireline stimulation is required. The assumption made is that tar is the part of non-movable heavy oil that does not show any mobility after thermal stimulation. If no mobility is detected by the formation tester after thermal stimulation, then most probably this non-movable part is tar. Side cores are required to further confirm this, as wireline stimulation techniques have a relatively short time span that might not be enough to stimulate heavy oil mobility.

The workflow described above shows how to identify tar in heavy oil yet it does not address the lateral extent of the tar zone. The NMR shallow depth of investigation makes it necessary to log each well drilled to try and map the tar zone. However, this is neither practical nor economical. To overcome NMR limitation, another technique to identify tar is required that can be run in most wells across the field. Our experience from geosteering shows that chemostratigraphy on drilling cuttings can be used to identify tar despite the measurement being non-sensitive to organic material. This is done by identifying a chemical finger print associated with tar during the well planning stage. The chemical finger print comprises a combination of elements that is unique to tar in a certain field. This tool was initially designed to help avoid tar zones while drilling. Since the measurement is made on drilling cuttings, it could be done on all drilled wells, whether observer, producer, or injector, regardless of whether wireline or logging while drilling information is available or not. 
Chemostratigraphy will allow identification of tar across the field. This information can be used as calibration points for any tar map.

Current surface seismic and borehole seismic tar mapping techniques can take advantage of both NMR based technique's, described above, high vertical resolution and chemostratigraphy's large number of calibration points to overcome limitation associated with seismic low vertical resolution. This can be done by linking tar identified in key wells where NMR, chemostratigraphy, and borehole seismic are available to seismic attributes that can be mapped. If 3D surface seismic is available the accuracy of such tar map will be much enhanced. The integrated solution described leads to more accurate estimates of reserves in heavy oil reservoirs. This will also help optimize reservoir management and investment in surface facilities. 